\title{
An unusual presentation of a giant pancreatic pseudocyst extending through the lateral abdominothoracic wall into the subcutaneous plane
}

\author{
Daniel Page*, Suresh Munugani
}

Department of General Surgery, Hervey Bay Hospital, Queensland, Australia

Received: 31 October 2020

Accepted: 09 November 2020

*Correspondence:

Dr. Daniel Page,

E-mail: danielpage26@me.com

Copyright: (C) the author(s), publisher and licensee Medip Academy. This is an open-access article distributed under the terms of the Creative Commons Attribution Non-Commercial License, which permits unrestricted non-commercial use, distribution, and reproduction in any medium, provided the original work is properly cited.

\begin{abstract}
Pancreatic pseudocysts are a common complication of pancreatitis affecting up to $40 \%$ of chronic cases. Typically, they are located within or abutting the pancreas and are self-limiting with the majority been smaller than $6 \mathrm{~cm}$, asymptomatic and resolving with conservative management. Here we present an unusual multiloculated giant pancreatic pseudocyst that originated at the pancreatic tail and extended through the lateral thoracoabdominal walls into the subcutaneous plane in a male with chronic alcohol pancreatitis. A literature search revealed no previous documented cases of this specific complication.
\end{abstract}

Keywords: Pancreatitis, Pseudocyst, Subcutaneous extension, Giant pseudocyst

\section{INTRODUCTION}

Pancreatic pseudocysts are a relatively common complication of pancreatitis affecting $10-26 \%$ of acute cases of pancreatitis and 20-40\% of chronic cases. ${ }^{1}$ Pseudocysts are fluid collections typically within or abutting the pancreas held within a sac created by fibrous granulation tissue lacking an epithelial lining; hence pseudocyst. The fluid is composed of pancreatic secretions as the collection is connected to the pancreatic ductal system.

Pseudocysts result from disruption to the pancreatic ductal system from increased pressure as a result of obstruction and necrosis secondary to pancreatitis with subsequent extravasation of pancreatic secretions that become walled off. ${ }^{2}$ The most common location of a pseudocyst is in the lesser sac with less common locations and extensions described including the mediastinum, the thigh and pleura. ${ }^{3,4}$

Here we present an unusual case of a giant multiloculated pseudocyst originating from the pancreatic tail and extending through the lateral thoracoabdominal walls into the subcutaneous plane.

A literature search revealed only one previously documented case of a pancreatic pseudocyst extending into the subcutaneous plane and this was a patient with extension through the lumbar musculature into the subcutaneous tissue of the lumbar back; making this case the first documented presentation of this specific complication. ${ }^{5}$

\section{CASE REPORT}

A 69-year-old male presented to the emergency department with an acute onset of left lateral chest wall pain, subjective fevers and associated increase in a lateral chest wall mass that had commenced 3 months earlier following a percutaneous drainage of a large left sided pleural effusion.

He had a previous history of alcohol related chronic pancreatitis with a first presentation approximately 2 years earlier to this presentation; serial imaging across this period demonstrated persistent pancreatic 
inflammation consistent with chronic pancreatitis and the eventual evolution of a multiloculated pseudocyst appearing on MRCP 16 months following his first episode of pancreatitis. In addition to this his Lipase had been persistently elevated in the 12 months preceding this episode measuring between $650-1200 \mathrm{U} / 1$ on multiple occasions.

Clinically he had a visible and palpable boggy swelling over the left lateral chest wall and flank (Figure 1) with a soft and nontender abdomen, he was afebrile, with normal respiratory and haemodynamic observations. His blood investigations demonstrated: WCC 15.3, CRP 220, bilirubin 12/8 $\mu \mathrm{mol} / \mathrm{l}$ (total/conjugated fraction), ALP $245 \mathrm{U} / \mathrm{l}$, GGT $122 \mathrm{U} / \mathrm{l}$, with normal transaminases, and lipase $920 \mathrm{U} / \mathrm{l}$. He had a contrast computed tomography (CT) of his chest, abdomen and pelvis, that showed a large multi loculated pseudocyst from the tail of the pancreas extending posterior-laterally towards the spleen and behind left kidney and superiorly into the left pleural cavity and also laterally through the thoraco-abdominal musculature into the lateral subcutaneous plane (Figure 2), his main pancreatic duct was noted to be dilated.

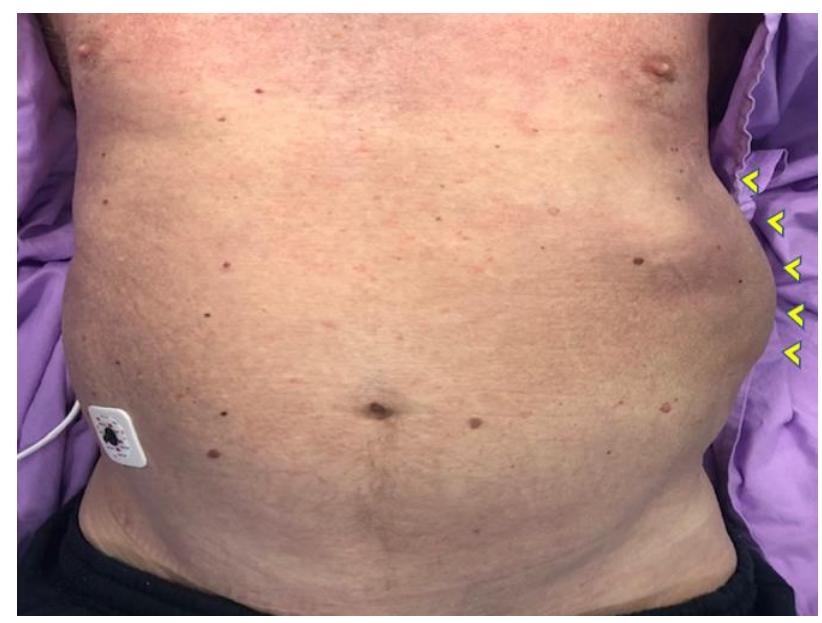

Figure 1: Clinical image of left sided boggy thoracoabdominal mass- outlined by yellow markers.

An ultrasound guided aspiration of this collection was performed, the fluid was noted to be dark, microscopy showed haemosiderin laden macrophages and inflammatory cells, culture was unremarkable and fluid lipase was $62,400 \mathrm{U} / 1$ consistent with a pseudocyst.

This gentleman was subsequently managed with a CT guided percutaneous drain into the collection with a second fluid sample sent that was similarly reflective of pseudocyst content and all cytology was negative for malignancy. Initially his symptoms of discomfort and the thoracoabdominal swelling improved with interval imaging also demonstrating significant regression in the size of the pseudocyst, he was discharge to home with his drain in situ. Unfortunately, he represented approximately 3 weeks later with further abdominal pain and repeat CT scan of his abdomen that showed he had developed a 26 mm splenic artery pseudoaneurysm that required coil embolization and subsequent splenectomy due to rupture.

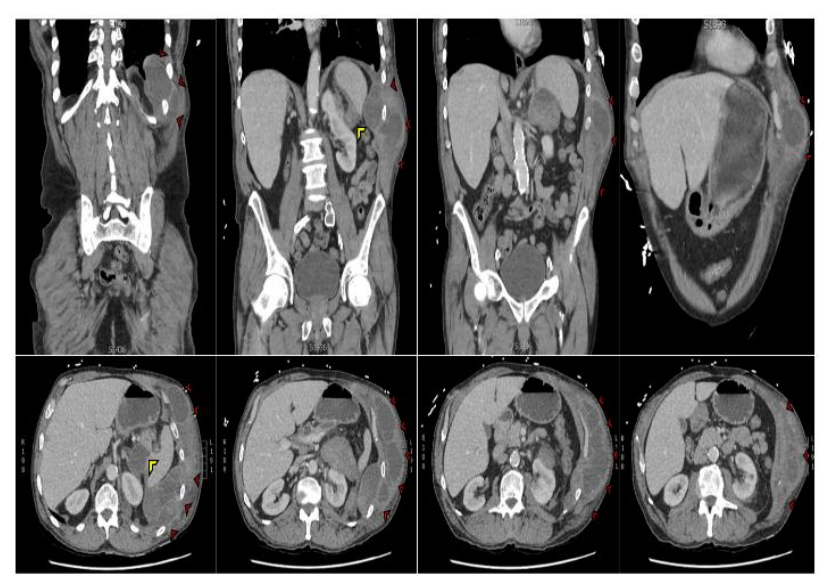

Figure 2: Contrast CT imaging with serial sections in the axial and coronal planes. Yellow arrows demonstrating origin of pseudocyst near pancreatic tail. Red arrows outlining pseudocyst extensions into multiple planes including into the pleural cavity, through the abdominothoracic musculature and into the subcutaneous plane.

\section{DISCUSSION}

Pancreatic pseudocysts are a relatively common complication of both acute and chronic pancreatitis and the vast majority that are $<4 \mathrm{~cm}$ will be asymptomatic and require no intervention and typically resolve without intervention. However, pseudocysts $>6 \mathrm{~cm}$ carry a higher risk of becoming symptomatic and requiring intervention. ${ }^{6,7}$

If symptomatic common symptoms are anorexia, abdominal discomfort, persistent pain, nausea and vomiting. Indications for intervention include persistent symptoms, infection, haemorrhage, persistent growth on serial imaging, pleural effusions or obstructive symptoms including gastric, duodenal, biliary and vascular obstruction.

Management options involve drainage of the pseudocyst which can take an endoscopic approach creating transpapillary, transgastric or transduodenal drainage routes; percutaneous drainage or surgical drainage. ${ }^{6,8}$

In this case the patient had an atypical presentation of a giant pancreatic pseudocyst $(>10 \mathrm{~cm}$ by definition) that unusually had penetrated through the thoracoabdominal musculature into the subcutaneous plane. This is one of only two documented cases of a pseudocyst extending into the subcutaneous plane to our knowledge and the first to extend into the anterolateral thoracoabdominal wall.

This patient met multiple criteria for percutaneous drainage including persistent expansion, infection and 
symptoms of a pseudocyst, in the absence of these it would have been safe to manage him expectantly. Unfortunately, he also suffered an additional pancreatitis complication of splenic artery aneurysm that required endovascular and subsequent surgical intervention due to rupture. He went on to make a good recovery post laparotomy and splenectomy.

\section{CONCLUSION}

Pancreatic pseudocysts are a common complication of pancreatitis but typically follow a benign and asymptomatic course. However, if expanding and are symptomatic then they require intervention. Typically, there are no gross clinical findings of a pancreatic pseudocyst, however they can become palpable if they are large and particularly if they have expanded into the subcutaneous plane, as in this instance. Although rare any patient presenting with a subcutaneous swelling with a background of pancreatitis should have an expanding pseudocyst considered as a differential and this is best defined with either contrast CT or magnetic resonance imaging.

Funding: No funding sources Conflict of interest: None declared

Ethical approval: Not required

\section{REFERENCES}

1. Samuerlson AL, Shah RJ. Endoscopic management of pancreatic pseudocysts. Gastroenterol Clin North Am. 2012;41(1):47-62
2. Habashi S, Draganov PV. Pancreatic pseudocyst. World J Gastroenterol. 2009;15(1):38-47.

3. Annangi S, Singh T, Maskey A, Gaurav K. Intrathoracic Extension of Pancreatic Pseudo Cyst: A Rare Cause of Hemoptysis. Chest. 2019;156(4):886.

4. Cierra C. Smith, DO, Alexander W. Smith, DO, Jay Anderson, DO. Mediastinal Pancreatic pseudocyst with expansion into bilateral pleural spaces. Am J Gastroenterol. 2019;114:S703-4

5. Fitchett JM, Beaumont A, Davies IL, Lewis MH. An extreme presentation of pancreatic pseudocyst. Ann R Coll Surg Engl. 2010;92(1):e21-3.

6. Lerch MM, Stier A, Wahnschaffe U, Mayerle J. Pancreatic pseudocysts: observation, endoscopic drainage, or resection? Deutsches Arzteblatt Int. 2009;106(38):614-21.

7. Udeshika WAE, Herath HM, Dassanayake SUB, Pahalagamage SP, Kulatunga A. A case report of giant pancreatic pseudocyst following acute pancreatitis: experience with endoscopic internal drainage. Brit Med Centr Res Notes. 2018;11:262.

8. Samuerlson AL, Shah RJ. Endoscopic management of pancreatic pseudocysts. Gastroenterol Clin North Am. 2012;41(1):47-62.

Cite this article as: Page D, Munugani S. An unusual presentation of a giant pancreatic pseudocyst extending through the lateral abdominothoracic wall into the subcutaneous plane. Int Surg J 2020;7:41613 . 